\title{
Identification of a novel large deletion of the mitochondrial DNA in an infant with Pearson syndrome: a case report
}

\author{
Rui Liu ${ }^{1}$, Gui-Ling $\mathrm{Mo}^{2}$, Yuan-Zong Song ${ }^{1}$ \\ ${ }^{1}$ Department of Pediatrics, The First Affiliated Hospital, Jinan University, Guangzhou, China; ${ }^{2}$ Guangzhou Kingmed Center for Clinical Laboratory, \\ Guangzhou, China \\ Correspondence to: Yuan-Zong Song, MD, PhD and Professor. Department of Pediatrics, The First Affiliated Hospital, Jinan University, Guangzhou \\ 510630, China. Email: songyuanzong@vip.tom.com.
}

\begin{abstract}
Pearson syndrome (PS), also known as Pearson marrow-pancreas syndrome, is a rare, multisystemic disorder caused by large-scale deletion of mitochondrial DNA (mtDNA) ranging from $2.3 \mathrm{~kb}$ to $9 \mathrm{~kb}$, with 4,977 bp in length as the most common variant. This paper reported a novel mtDNA deletion of 4,734 bp in size, spanning from nucleotide 11,220 to 15,953 . The infant suffered from chronic hepatomegaly, liver dysfunction, anemia and lactic acidosis over 1 year. Evidences of any infections were negative. Bone marrow aspiration and whole exome sequencing covering nearly 20,000 nucleus genes were performed when aged 3.3 and 6 months, respectively, but no genetic cause was identified. However, at his age 13 months, multiplex ligation-dependent probe amplification assay of the mtDNA in the patient detected a large deletion of 4,734 bp in size spanning the mitochondrial genes MTND4, MTTH, MTTS2, MTTL2, MTND5, MTND6, MTTE, MTCYB and MTTT which were functionally crucial for the intact oxidative phosphorylation pathway and adenosine triphosphate production, and PS was thus definitely diagnosed. This large deletion was negative in his parents and elder brother. Oral ursodeoxycholic acid, fat-soluble vitamins and blood transfusions were administrated, his clinical and laboratory presentations remained stable so far, but the long-term prognosis needed to be followed up. These findings enriched the variant spectrum of mtDNA, and demonstrated the importance of considering mitochondrial disorder in patient with intractable anemia, liver dysfunction and lactic acidosis as well as the significance of appropriate choosing of relevant genetic tools in the etiology diagnosis of such patients.
\end{abstract}

Keywords: Infant; mitochondrial DNA (mtDNA); Pearson syndrome (PS); variant; case report

Submitted May 08, 2020. Accepted for publication Nov 25, 2020.

doi: $10.21037 /$ tp-20-138

View this article at: http://dx.doi.org/10.21037/tp-20-138

\section{Introduction}

Pearson syndrome (PS) first described by Pearson and his colleagues in 1979 (1), is a rare, multi-systemic mitochondrial disorder that occurs in early infancy, featured by vacuolated marrow precursors and exocrine pancreatic dysfunction (2). Other clinical presentations include lactic acidosis, insulin-dependent diabetes mellitus, liver dysfunction, renal tubular dysfunction, failure to thrive, and muscular and neurologic impairment (3-6). PS is caused by single large-scale mitochondrial DNA (mtDNA) deletions (SLSMDs) ranging from 2.3 to $9 \mathrm{~kb}$, with $4,977 \mathrm{bp}$ in length as the most common variant (7). Due to one or multiple mtDNA deletions of varying size, location and proportion, mitochondrial oxidative phosphorylation (OXPHO) could be impaired, resulting in reduced energy production (8). The diagnosis of PS syndrome depends on mtDNA analysis (7). To the best of our knowledge, the reported PS cases were rather limited in number, and up to date, about 109 PS patients were reported, including 52 ones with infant onset $(6,9-12)$. Here, we reported a rare and challenging case with atypical clinical presentation soon after birth, which was definitely diagnosed by mtDNA analysis. These findings also enriched the variant spectrum of mtDNA. In combination with the 
Table 1 Laboratory indices over time in the patient

\begin{tabular}{|c|c|c|c|c|c|c|c|c|c|}
\hline Indices (reference range) & \multicolumn{9}{|c|}{ Ages } \\
\hline ALT (5-40 U/L) & - & 24 & - & - & 42 & - & 106 & 131 & 107 \\
\hline AST (8-40 U/L) & - & 60 & 119 & 107 & 120 & 92 & 112 & 105 & 180 \\
\hline GGT (7-50 U/L) & - & 157 & 759 & 248 & - & 94 & 251 & 319 & 352 \\
\hline TBIL (2-17 ㅆmol/L) & 216.9 & 260.7 & 70.6 & 232.5 & 163.9 & 185.0 & - & 9.6 & 17.7 \\
\hline DBIL (0-7 $\mu \mathrm{mol} / \mathrm{L})$ & - & 22.3 & 24.2 & 165.7 & 120.6 & 123.3 & - & 3.7 & 8.8 \\
\hline IBIL (2-13.7 $\mu \mathrm{mol} / \mathrm{L})$ & - & 238.4 & 46.4 & 66.8 & - & 62.6 & - & 5.9 & 8.9 \\
\hline $\operatorname{RBC}\left[(4-4.5) \times 10^{12} / L\right]$ & - & 3.35 & 2.43 & 2.59 & 2.26 & 3.57 & 2.80 & 2.72 & 2.88 \\
\hline
\end{tabular}

$\mathrm{D}$, day; M, month; ALT, alanine transaminase; AST, aspartate transaminase; TBA, total bile acids; GGT, gamma-glutamyl transpeptidase; TBIL, total bilirubin; DBIL, direct bilirubin; IBIL, indirect bilirubin; RBC, red blood cells; HGB, hemoglobin.

patient's clinical manifestations, early etiology exploration was important for the diagnosis and management of such patients.

We presented the following case in accordance with the CARE reporting checklist (13). Available at http://dx.doi. org/10.21037/tp-20-138.

\section{Case presentation}

A 13-month-old male patient was referred to our clinic due to hepatomegaly, abnormal liver function and anemia discovered over 1 year. At the age of 18 hours, he was referred to neonatal intensive care unit due to jaundice and tachypnea soon after birth. The liver was normal in size and texture, but the serum levels of total bile acids, total bilirubin, aspartate transaminase and lactate were all elevated, along with a hemoglobin level of $91 \mathrm{~g} / \mathrm{L}$ (Table 1). The diagnoses of moderate anemia, liver dysfunction and lactic acidosis were thus made while phototherapy and blood transfusion were given. As a result, jaundice and anemia were alleviated, but abnormal liver function and lactic acidosis remained refractory. Since being discharged at age 1.5 months, he was referred to different hospitals due to persisted abnormal liver function, intractable anemia and lactic acidosis despite the usage of oral ursodeoxycholic acid (5 mg/kg, q12h), coenzyme Q (10 mg, qd), carnitine (50 mg/kg, qd) and vitamin D (400 IU, qd). The patient became transfusion dependent for red blood cells and he developed transient neutropenia in the first 2 months of life (minimum $0.83 \times 10^{9} / \mathrm{L}$ ). Evidences of any infections were negative. Bone marrow aspiration (BMA) and whole exome sequencing (WES) covering nearly 20,000 nucleus genes were performed when aged 3.3 and 6 months, respectively, but no genetic cause was identified.

As the second product of a non-consanguineous couple, the child was delivered spontaneously at the gestational age of 39 weeks after an uneventful pregnancy, with a birth weight of $3.2 \mathrm{~kg}$, body length $51 \mathrm{~cm}$ and head circumference $34 \mathrm{~cm}$. The parents were healthy, and there was no family history of any genetic or metabolic disorders.

Physical examination at referral revealed a body weight of $7.4 \mathrm{~kg}$ [<-3 standard deviation (SD)], height $74.8 \mathrm{~cm}$ $(<-3 \mathrm{SD})$, and head circumference $42.9 \mathrm{~cm}(<-2 \mathrm{SD})$. No cutaneous and scleral pallor or jaundice could be observed. The lungs were clear. No murmurs or abnormal heart sounds were heard. There was no abdominal distention, but a liver $3.5 \mathrm{~cm}$ below the right subcostal margin with medium texture was palpable. The spleen was not enlarged. Physiological reflexes were normal and no pathological reflexes could be found on nervous system examination. The extremities were warm, and the distal perfusion was excellent.

Biochemistry tests revealed abnormal liver function and lactic acidosis (Table 1). Multiplex ligation-dependent probe amplification (MLPA) assay of the mtDNA detected a large deletion involving 9 mitochondrial genes including MTND4, MTTH, MTTS2, MTTL2, MTND5, MTND6, 


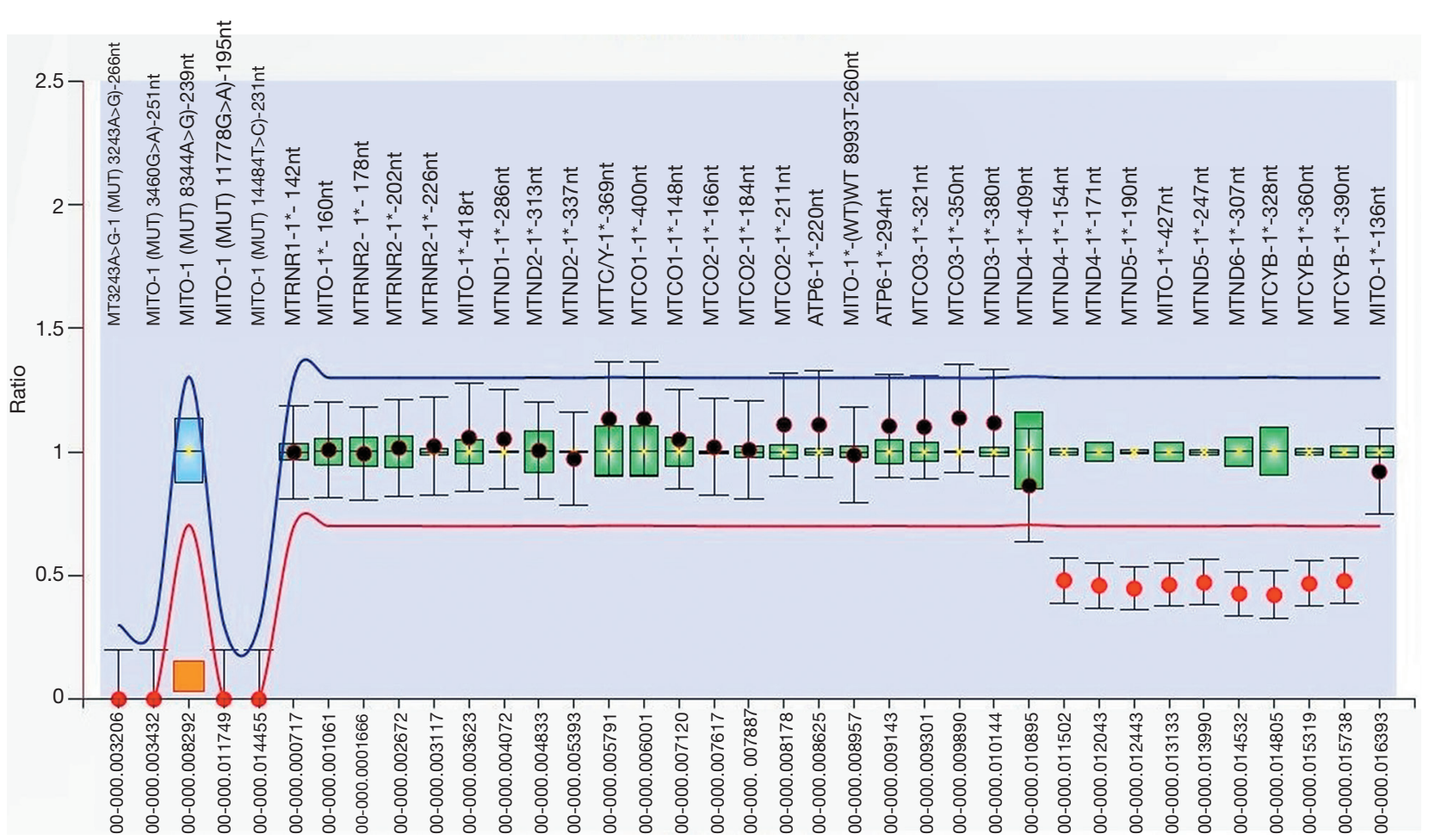

Figure $1 \mathrm{MLPA}$ assay results of the patient. The horizontal axis represented the probe positions, while the vertical, the fluorescence signal ratio of subject sample/normal control which was represented as mean \pm SD in this figure. On the top were the probe labels. The red and the blue curves represented the normal upper and lower limits of the ratios, respectively. The green boxes indicated the reliability of the experiments. The 9 genes MTND4, MTTH, MTTS2, MTTL2, MTND5, MTND6, MTTE, MTCYB and MTTT (dots in red on the right) displayed ratios ranging from 0.25 to 0.75 , indicating a large deletion. The remaining 23 mtDNA genes (dots in black) exhibited normal ratios. MLPA, multiplex ligation-dependent probe amplification; SD, standard deviation.

MTTE, MTCYB and MTTT in the patient (Figure 1), which was negative in his parents and elder brother. Further nextgeneration sequencing (NGS) test of the mitochondrial genome uncovered a mtDNA deletion of $4,734 \mathrm{bp}$ (from nucleotide 11,220 to 15,953 ), confirming the MLPA findings. Figure 2 was the timeline of the relevant information about the patient.

PS was thus definitely diagnosed, and oral vitamin D (400 IU, qd) was suggested to be remained. He had normal developmental milestones, his physical, cognitive, social, and language development have remained typical for age. His condition remained stable so far, but he still suffered from mild anemia, abnormal liver function and lactic acidosis, and clinic follow up was underway.

All procedures performed in this study were in accordance with the ethical standards of the institutional and/or national research committee(s) and with the Helsinki Declaration (as revised in 2013). Written informed consent was obtained from the parents.

\section{Discussion}

The diagnosis of PS in newborns is often difficult, because its clinical features may overlap with infectious, neuromuscular, hematological, metabolic, or digestive diseases $(8,14)$. Hepatomegaly, liver dysfunction, anemia and lactic acidosis were chronic but non-pathognomonic presentations in this patient. Although a series of tests including WES analysis had been conducted, the etiology remained unclear until MLPA revealed the novel mtDNA deletion which was confirmed by NGS analysis. This child's case enriched the mtDNA mutation spectrum and supported the complexity of the etiology exploration process as well as the significance of appropriate choosing of relevant analytic tools for genetic diseases.

The genes ND4, ND5 and ND6 encoded subunits of complex I while CYB encoded a subunit of complex III in the mitochondrial respiratory chain (MRC) (15), and thus deletion in this patient affected MRC to produce adenosine triphosphate (ATP). The MRC impairment led to disruption 


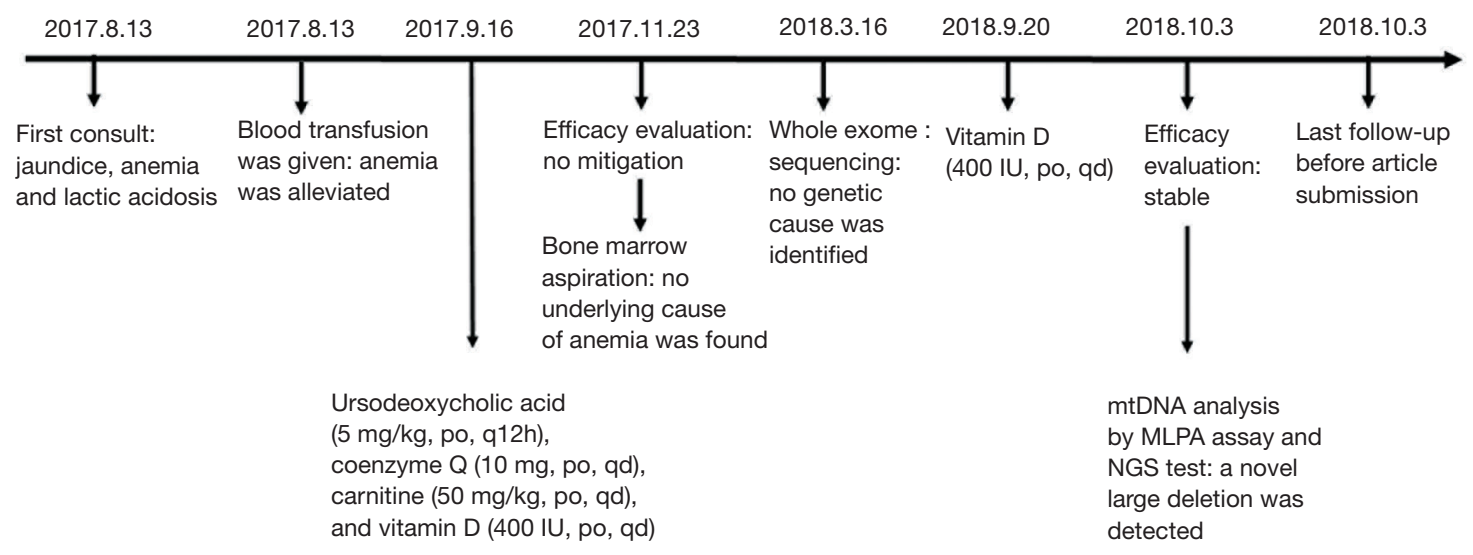

Figure 2 Timeline of the patient.

of Krebs cycle and thus accumulation of pyruvate, which was converted to lactate, giving rise to his intractable lactic acidosis (16). Due to the mtDNA mutation, an erythroid maturation was defected (17), constituting an explanation for his prolonged anemia. Meanwhile, ATP shortage in the hepatocyte hampered the canalicular carrier proteins to transport bile components like bile acids and bilirubin, contributing to his hepatomegaly and liver dysfunction (18).

There is no curative treatment for PS patients and therapy is primarily symptomatic. Blood transfusion and bicarbonate supplementation was required for management. And pancreatic enzyme replacement is needed for patients with malabsorption due to exocrine pancreatic dysfunction. The prognosis of PS patient was poor usually, and about $75 \%$ of them died before 5 years of age, with intractable metabolic acidosis, renal or hepatic failure and sepsis (7). Although stable in condition currently, the long-term outcome of our patient needs to be observed.

This paper described the clinical and molecular presentations of a pediatric PS patient harboring a large mtDNA deletion. For the well understanding of the genetic and phenotypic features of this rare mitochondrial disorder, however, more PS patients need to be identified and characterized.

\section{Conclusions}

In conclusion, this paper reported an infant with PS caused by a novel mtDNA deletion of 4,734 bp. These findings enriched the variant spectrum of mtDNA, and demonstrated the importance of considering mitochondrial disorder in patient with intractable anemia, liver dysfunction and lactic acidosis as well as the significance of appropriate choosing of relevant genetic tools in the etiology diagnosis of such patients.

\section{Acknowledgments}

The authors were deeply grateful to the PS patient and his family members for their cooperation.

Funding: This paper was supported financially by National Natural Science Foundation of China (NSFC, No. 81974057).

\section{Footnote}

Reporting Checklist: The authors have completed the CARE reporting checklist. Available at http://dx.doi.org/10.21037/ tp-20-138

Peer Review File: Available at http://dx.doi.org/10.21037/ tp-20-138

Conflicts of Interest: All authors have completed the ICMJE uniform disclosure form (available at http://dx.doi. org/10.21037/tp-20-138). The authors have no conflicts of interest to declare.

Ethical Statement: The authors are accountable for all aspects of the work in ensuring that questions related to the accuracy or integrity of any part of the work are appropriately investigated and resolved. All procedures performed in this study were in accordance with the ethical standards of the institutional and/or national research committee(s) and with the Helsinki Declaration (as revised in 2013). Written informed consent was obtained from the parents. 
Open Access Statement: This is an Open Access article distributed in accordance with the Creative Commons Attribution-NonCommercial-NoDerivs 4.0 International License (CC BY-NC-ND 4.0), which permits the noncommercial replication and distribution of the article with the strict proviso that no changes or edits are made and the original work is properly cited (including links to both the formal publication through the relevant DOI and the license). See: https://creativecommons.org/licenses/by-nc-nd/4.0/.

\section{References}

1. Pearson HA, Lobel JS, Kocoshis SA, et al. A new syndrome of refractory sideroblastic anemia with vacuolization of marrow precursors and exocrine pancreatic dysfunction. J Pediatr 1979;95:976-84.

2. Rötig A, Cormier V, Blanche S, et al. Pearson's marrowpancreas syndrome. A multisystem mitochondrial disorder in infancy. J Clin Invest 1990;86:1601-8.

3. Rötig A, Bourgeron T, Chretien D, et al. Spectrum of mitochondrial DNA rearrangements in the Pearson marrowpancreas syndrome. Hum Mol Genet 1995;4:1327-30.

4. Atale A, Bonneau-Amati P, Rötig A, et al. Tubulopathy and pancytopaenia with normal pancreatic function: a variant of Pearson syndrome. Eur J Med Genet 2009;52:23-6.

5. Chen XY, Zhao SY, Wang Y, et al. A novel mitochondrial DNA deletion in a patient with Pearson syndrome and neonatal diabetes mellitus provides insight into disease etiology, severity and progression. Mitochondrial DNA A DNA Mapp Seq Anal 2016;27:2492-5.

6. Wild KT, Goldstein AC, Muraresku C, et al. Broadening the phenotypic spectrum of Pearson syndrome: Five new cases and a review of the literature. Am J Med Genet A 2020;182:365-73.

7. Farruggia P, Di Marco F, Dufour C. Pearson syndrome. Expert Rev Hematol 2018;11:239-46.

8. Manea EM, Leverger G, Bellmann F, et al. Pearson syndrome in the neonatal period: two case reports and review of the literature. J Pediatr Hematol Oncol 2009;31:947-51.

9. Pronman L, Rondinelli M, Burkardt DD, et al. Pearson Syndrome: A Rare Cause of Failure to Thrive in Infants.

Cite this article as: Liu R, Mo GL, Song YZ. Identification of a novel large deletion of the mitochondrial DNA in an infant with Pearson syndrome: a case report. Transl Pediatr 2021;10(1):204-208. doi: 10.21037/tp-20-138
Clin Pediatr (Phila) 2019;58:819-24.

10. Vadhul R, Halbach CS, Areaux RG Jr, et al. Endothelial dysfunction in a child with Pearson marrow-pancreas syndrome managed with Descemet stripping automated endothelial keratoplasty using a suture pull-through technique. Digit J Ophthalmol 2019;25:59-64.

11. Jennifer MS, Cortez D. Pearson marrow-pancreas syndrome with cardiac conduction abnormality necessitating prophylactic pacemaker implantation. Ann Noninvasive Electrocardiol 2020;25:e12681.

12. Gustafson MA, McCormick EM, Perera L, et al. Mitochondrial single-stranded DNA binding protein novel de novo SSBP1 mutation in a child with single largescale mtDNA deletion (SLSMD) clinically manifesting as Pearson, Kearns-Sayre, and Leigh syndromes. PLoS One 2019;14:e221829.

13. Riley DS, Barber MS, Kienle GS, Aronson JK, von Schoen-Angerer T, Tugwell P, Kiene H, Helfand M, Altman DG, Sox H, Werthmann PG, Moher D, Rison RA, Shamseer L, Koch CA, Sun GH, Hanaway P, Sudak NL, Kaszkin-Bettag M, Carpenter JE, et al. CARE guidelines for case reports: explanation and elaboration document. J Clin Epidemiol 2017;89:218-35.

14. Tadiotto E, Maines E, Degani D, et al. Bone marrow features in Pearson syndrome with neonatal onset: A case report and review of the literature. Pediatr Blood Cancer 2018;65:1-4.

15. Wallace DC. Mitochondrial genetic medicine. Nat Genet 2018;50:1642-9.

16. Farruggia P, Di Cataldo A, Pinto RM, et al. Pearson Syndrome: A Retrospective Cohort Study from the Marrow Failure Study Group of A.I.E.O.P. (Associazione Italiana Emato-Oncologia Pediatrica). JIMD Rep 2016;26:37-43.

17. Ahlqvist KJ, Leoncini S, Pecorelli A, et al. MtDNA mutagenesis impairs elimination of mitochondria during erythroid maturation leading to enhanced erythrocyte destruction. Nat Commun 2015;6:6494.

18. Chen HL, Wu SH, Hsu SH, et al. Jaundice revisited: recent advances in the diagnosis and treatment of inherited cholestatic liver diseases. J Biomed Sci 2018;25:75. 